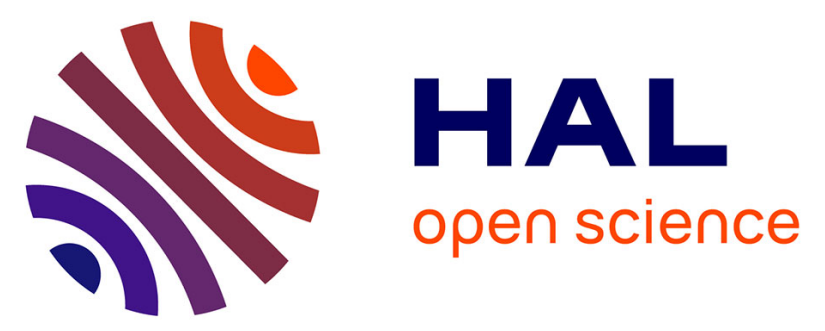

\title{
Nanodisc-Based Bioelectronic Nose Using Olfactory Receptor Produced in Escherichia coli for the Assessment of Death-Associated Odor Cadaverine
}

Heehong Yang, Daesan Kim, Jeongsu Kim, Dongseok Moon, Hyun Seok Song, Minju Lee, Seunghun Hong, Tai Hyun Park

\section{To cite this version:}

Heehong Yang, Daesan Kim, Jeongsu Kim, Dongseok Moon, Hyun Seok Song, et al.. Nanodisc-Based Bioelectronic Nose Using Olfactory Receptor Produced in Escherichia coli for the Assessment of DeathAssociated Odor Cadaverine. ACS Nano, 2016, 11 (12), pp.11847-11855. 10.1021/acsnano.7b04992 . hal-02388831

\section{HAL Id: hal-02388831 \\ https://hal.science/hal-02388831}

Submitted on 2 Dec 2019

HAL is a multi-disciplinary open access archive for the deposit and dissemination of scientific research documents, whether they are published or not. The documents may come from teaching and research institutions in France or abroad, or from public or private research centers.
L'archive ouverte pluridisciplinaire $\mathbf{H A L}$, est destinée au dépôt et à la diffusion de documents scientifiques de niveau recherche, publiés ou non, émanant des établissements d'enseignement et de recherche français ou étrangers, des laboratoires publics ou privés. 


\section{Nanodisc-Based Bioelectronic Nose Using}

\section{Olfactory Receptor Produced in Escherichia coli}

\section{for the Assessment of Death-Associated Odor}

\section{Cadaverine}

Heehong Yang ${ }^{a, t}$, Daesan Kim ${ }^{b, t}$, Jeongsu Kim ${ }^{c}$, Dongseok Moon ${ }^{a}$, Hyun Seok Song ${ }^{d, e}$, Minju Lee ${ }^{c}$, Seunghun Hong, ${ }^{c, *}$ and Tai Hyun Park ${ }^{a, *}$

${ }^{\mathrm{a}}$ School of Chemical and Biological Engineering, Seoul National University, Seoul 08826, Republic of Korea

${ }^{\mathrm{b}}$ Department of Biophysics and Chemical Biology, Seoul National University, Seoul 08826, Republic of Korea

${ }^{c}$ Department of Physics and Astronomy and Institute of Applied Physics, Seoul National University, Seoul 08826, Republic of Korea

${ }^{\mathrm{d}}$ Division of Bioconvergence Analysis, Korea Basic Science Institute (KBSI), Daejeon 34133, Republic of Korea

${ }^{\mathrm{e}}$ Center for Convergent Research of Emerging Virus Infection, Korea Research Institute of Chemical Technology, Daejeon 34114, Republic of Korea 
*To whom correspondence should be addressed.

E-mail: thpark@ snu.ac.kr (T.H. Park), seunghun@snu.ac.kr (S. Hong)

RECEIVED DATE (to be automatically inserted after your manuscript is accepted if required according to the journal that you are submitting your paper to)

ABSTRACT: Cadaverine (CV), a death-associated odor, is an important target molecule for various sensor applications, including the evaluation of food spoilage. In this study, we developed an oriented ND-functionalized bioelectronic nose $(\mathrm{ONBN})$, based on carbon nanotube transistors and nanodiscs (NDs) embedded with an olfactory receptor (OR) produced in Escherichia coli (E. coli) for detection of CV. To fabricate ONBN devices, a trace amine-associated receptor $13 \mathrm{c}$ (TAAR13c) binding to $\mathrm{CV}$ was produced in E. coli, purified, reconstituted into NDs and assembled, in the desired orientation, onto a carbon nanotube (CNT)-based field effect transistor with floating electrodes. The ONBN showed high performance in terms of sensitivity and selectivity. Moreover, the ONBN was used to measure CV in diverse real-food samples for the determination of food freshness. These results indicate ONBN devices can be utilized to evaluate the quality of food samples quantitatively, which should enable versatile practical applications such as food safety and preservative development. Moreover, the ONBN could provide a useful tool for detection of corpses, which could be practically used in disaster responses.

\section{KEYWORDS}

nanodiscs, bioelectronic nose, field-effect transistor, trace amine-associated receptor, cadaverine 
G protein-coupled receptors (GPCRs) play important roles in the cellular responses of the human body. Thus, they are significantly involved in many human diseases and are the target of approximately $40 \%$ of all modern medicinal drugs. ${ }^{1,2}$ Trace amine-associated receptors (TAARs), a class of GPCR, are conventional amine receptors binding to endogenous compounds structurally related to classical biogenic amines. It has been reported that trace amine-associated receptor 13c (TAAR13c) in the zebrafish (Danio rerio) functions as an olfactory receptor (OR) and has an efficient specificity to the death-associated odor cadaverine $(\mathrm{CV}) .^{3-6} \mathrm{CV}$, generated by bacterial decarboxylation of lysine, is an extremely repulsive odor to humans, among various biogenic amines. In addition, $\mathrm{CV}$ is one of the important markers for decayed foods because various kinds of food products contain lysine. Therefore, it has been suggested that the detection of CV can be applied to various fields of industrial applications and scientific investigations. ${ }^{3,7}$

For the production of recombinant proteins, the Escherichia coli (E. coli) has been widely used as a host cell because of its great advantage in productivity and convenience. However, the production of GPCR in E. coli remains a challenge because of their strong hydrophobicity, a complicated charge distribution and membrane inserting mechanism of bacterial cells. ${ }^{8-10}$ Among many reconstitution techniques of receptors, nanodiscs (NDs) have been considered the most appropriate tool for GPCR reconstitution. ${ }^{11,12}$ NDs are composed of a receptor, a lipid bilayer and membrane scaffold proteins (MSPs), which tightly wrap the edge of the a lipid bilayer, thus NDs can be stable in an aqueous environment ${ }^{10,12-14}$ and mimic the native structure of the receptor in a cell. ${ }^{13,14}$ An ND-based biosensor using Sf9 insect cells has also been reported. ${ }^{13}$ However, an ND-based biosensor using an E. coli system, which has great advantages in productivity over other systems, has not been previously reported. A carbon nanotube field-effect transistor (CNT-FET) with floating electrodes can exhibit improved 
performance compared with a conventional CNT-FET. ${ }^{15,16}$ The floating electrode structure on a CNT-FET can enhance the sensitivity of the device via the increased effect of Schottky barrier modulation. The Au floating electrodes also have advantages in the functionalization of specific biomolecules on their surfaces. The surfaces of floating electrodes have larger areas than conventional devices as well as more suitable conditions for the functionalization of biomolecules.

Here, we report the development of an oriented nanodisc-functionalized bioelectronic nose (ONBN) using TAAR13c-embedded nanodiscs (T13NDs) with high selectivity and sensitivity for the detection of CV. The T13NDs using receptors produced in E. coli enable the immobilized receptor to have stability and a natural binding pocket. Moreover, the T13NDs were immobilized on floating electrodes of a CNT-FET in a desired orientation, which increased the total binding sites of T13NDs. As a result, the ONBN exhibited improved performance in terms of sensitivity and selectivity, as well as reliability and reproducibility. Furthermore, the ONBN could distinguish the target molecules in food samples with differing degrees of spoilage. Our approach can offer a useful sensing system toward a practical bioelectronic sensor to enable the detection of death-associated compounds.

\section{RESULTS AND DISCUSSION}

Figure 1 depicts the schematic diagram and the predicted electrical response of an ONBN. A CNT-FET with floating electrodes was fabricated following previously-reported processes. A detailed method is described in Supporting Information (Figure S1). In brief, CNTs were patterned on a $\mathrm{SiO}_{2}$ substrate as transistor channels, and then, $\mathrm{Pd} / \mathrm{Au}$ electrodes were fabricated via thermal evaporation method. Finally, the source and drain electrodes were passivated using a photoresist to avoid a current leakage in an aqueous environment. After 
the fabrication of the device, T13NDs were immobilized on the Au surfaces of the floating electrodes. In this research, we used the half V5 antibody (Ab) as linker molecules for oriented immobilization of T13NDs on gold floating electrode. It has been reported that halffragment antibodies can be immobilized onto the gold surfaces with an orientation via their native thiol groups. ${ }^{17}$ The receptor TAAR13c has a V5 epitope at C-terminal. Therefore, the T13NDs can be immobilized with the desired orientation using half-fragment V5 Ab as a linker. Here, the V5 epitope of T13NDs binds to the half-fragment V5 Ab on the Au surface. The immobilized T13NDs on the Au surfaces were oriented such that the binding pockets of TAAR13c on the device were on the exposed side. In previous work, devices with randomly immobilized biomolecules also have been reported. ${ }^{18,19}$ However, randomly oriented sensors have a limited capability to bind target molecules. Therefore, ONBNs could provide an ideal method for the detection of specific target molecules.

We used a dual-glo luciferase assay system, which was suitable for the characterization of the TAAR13c in terms of its selectivity, to measure the dose-dependent response of TAA13c expressed in HEK-293 cells (Figure 2a). ${ }^{20}$ TAAR13c-expressed cells exhibited a significant response to $\mathrm{CV}$, but mock vector-transfected cells had no meaningful responses. This supports the conclusion that TAAR13c was successfully expressed in HEK-293 cells (Figure $2 \mathrm{a}, \mathrm{S} 2 \mathrm{a})$. Figure $2 \mathrm{~b}$ shows the selective responses of TAAR13c to $1 \mu \mathrm{M}$ stimulus of various amines. TAAR13c was co-expressed with RTP1S and $\mathrm{G}_{\text {oolf }}$, increasing the level of expression and efficiency of signal transduction. The selective binding properties of TAAR13 were investigated using various amines, which had different amine moieties and structures (Figure S2b). Our results show TAAR13c responded selectively to CV among various amines. Although putrescine (PT) appeared to stimulate the TAAR13c partially, there were no 
significant responses. The results are consistent with previous reports that TAAR13c is the most sensitive and selective receptor to $\mathrm{CV} .{ }^{3,5}$

Toward a receptor-based research, the TAAR13c expressed in E. coli (Figure 2c) was purified with a high purity and applied to the formation of receptor NDs. The purification and functional reconstitution of GPCRs for the development of receptor-based biosensors were reported previously. ${ }^{10,21}$ For the production of T13NDs, TAAR13c and lipids were mixed as detergent micelles, and then the membrane scaffold protein apolipoprotein A-I (ApoA-I) was added to enclose the lipid/receptor complex. The T13NDs were finally obtained after the removal of detergent using Bio-bead. The bands of TAAR13c in gel staining indicate that TAAR13c was purified with a high purity. Moreover, the observed bands were successfully confirmed as TAAR13c by a western blot analysis using V5 epitope Ab, confirming that TAAR13c was prepared with a high quality.

Dynamic light scattering (DLS) measurement of T13NDs (Figure 2d) indicated that size distribution of optimized T13NDs. For the assembly of the T13NDs, ApoA-I, the representative membrane scaffold protein, was successfully expressed in E. coli and purified with a high purity (Figure S3). After the assembly of the NDs, the ND-complex solutions were subjected to size exclusion chromatography (SEC) for the removal of residual unbound proteins (Figure S4). The fraction of T13NDs from the SEC separation was collected and analyzed by DLS. ${ }^{22}$ We have tested various conditions, such as lipid sonication time and protein concentration for the formation of receptor-NDs and found the best choice for the formation of optimized T13NDs (Figure S5). This results in a quite narrow size distribution of T13NDs with ca. $20 \mathrm{~nm}$ in diameter (Figure 2d), which is close to the diameter values estimated by FE-SEM images (Figure 2e). These results clearly demonstrate that the T13NDs were successfully self-assembled in homogeneous discoidal shapes. 
In order to analyze the affinity of T13NDs with $\mathrm{CV}$, the real-time tryptophan fluorescence of T13NDs was measured (Figure 2f). With the sequential addition of increasing CV amounts up to $7 \mathrm{mM}$, a significant fluorescence change of T13NDs was displayed. On the other hand, there was no response to the control buffer solution. The T13NDs responded selectively to $\mathrm{CV}$ in real-time tryptophan fluorescence (Figure $2 \mathrm{~g}$ ) in comparison with various amines which have different structures and amine moieties. The intrinsic tryptophan fluorescence of T13NDs was significantly quenched only by a stimulus of CV. Many researches have reported that the intrinsic fluorescence of functional receptor is quenched by selective binding of a ligand to the receptor. ${ }^{10,23,24}$ Therefore, these results show that the TAAR13c was effectively reconstituted in NDs.

Figure 3 shows the characteristics of an ONBN. Figure 3 a shows the fluorescence image of V5 Ab-functionalized floating electrodes and its intensity profile graph. To prepare T13NDs layers with the desired orientation, we utilized V5 Ab as linker molecules to attach T13NDs to the floating electrode surfaces. Then, the V5-functionalized Au electrodes were incubated in fluorescence dye-labeled V5 Ab solution (Detailed methods in METHODS section) and fluorescence microscopy was utilized to confirm functionalization of V5 Ab on floating electrodes of the ONBN. The image clearly shows the bright regions corresponding to the floating electrodes functionalized with $\mathrm{V} 5 \mathrm{Ab}$, while the dark regions are the $\mathrm{SiO}_{2}$ surfaces. Note that the intensity profile of the $\mathrm{Au}$ surface was higher than that of the $\mathrm{SiO}_{2}$ surface as shown in the intensity profile (bottom of Figure 3a). This result indicates that V5 Ab was successfully immobilized on the floating electrodes.

Atomic force microscopy (AFM) images of the T13ND-functionalized floating electrode in liquid environments and corresponding height profile (Figure 3b) shows functionalization of T13NDs on the floating electrode. The left and right images were measured in a tapping 
mode AFM with scan rates of 0.4 and $0.1 \mathrm{~Hz}$, respectively. The bright or dark regions correspond to T13ND-functionalized floating electrode or CNT channels on $\mathrm{SiO}_{2}$ substrates. We can find white spots with their heights of $\sim 10 \mathrm{~nm}$ correspond to the previously reported height of NDs. ${ }^{11,25}$ Here, because of tip convolution effects, the image of $\mathrm{x}-\mathrm{y}$ dimension is broader than the original one. ${ }^{26,27}$ This result implies the functionalization of T13NDs on the floating electrode. The immobilization of half-V5 Ab using disulfide bonding between the thiol groups of a functionalized Au surface and half-V5 Ab was confirmed via fluorescent microscopy (Figure 3a). Also, the height of T13NDs was similar with previously reported result (Figure 3b). These results clearly indicate that the T13NDs were assembled on the floating electrodes via antibody-antigen interaction which is the highly specific binding reaction. Therefore, we can conclude that T13NDs have the specific orientation on the Au surfaces.

The gate profiles of an ONBN before and after the immobilization of T13NDs (Figure 3c) exhibited typical p-type semiconductor characteristics in both cases, indicating that the transistor characteristics of the ONBN was maintained even after the immobilization of T13NDs on floating electrodes. Note that the electrical currents were reduced by the immobilization of T13NDs. This is presumably because negatively-charged parts of receptors immobilized on floating electrodes caused the gating effect and affected the drain-source currents. $^{28}$

The electrical noise characteristics of an ONBN (Figure 3d) could be fitted by a straight line, indicating a typical 1/f noise behavior. The $\mathrm{y}$-axis $\left(\mathrm{S} / \mathrm{I}^{2}\right)$ of graph means the noise power according to the change of current level versus the frequency. The noise amplitude was found to be $\sim 10^{-6}$ which was similar to the previously-reported values of clean network-based CNT-FETs without T13NDs. ${ }^{29,30}$ These results indicate that the functionalization of T13NDs 
on our CNT devices did not increase its noise levels, which can be critical in achieving a high sensitivity in our devices.

Figure 4a shows the real-time response of an ONBN to various concentrations of CV. Here, while adding various concentrations of target solutions, we measured drain-source currents of the ONBN using a semiconductor analyzer. The drain-source bias voltage was maintained as $0.1 \mathrm{~V}$ during the measurement. To monitor the response of our ONBNs to $\mathrm{CV}$, we develop the buffer zone of $9 \mu \mathrm{L}$ HEPES solution. After $100 \mathrm{~s}$, we consecutively added $10 \mathrm{pM} \mathrm{CV}$ solution of $1 \mu \mathrm{L}$ onto the buffer zone. In a similar manner, CV solutions were introduced from a low concentration to a high concentration by the factor of 10 at each time. The concentrations of added solutions were calculated by considering the increased solution volume. Then, the measured data of current changes during the additions of $\mathrm{CV}$ were normalized by the original current values to estimate the normalized conductance change $\Delta G / G_{0}$. Note that the conductance of the ONBN was sharply increased after adding various concentrations of CV solutions. The ONBN exhibited increased conductance by CV solutions with concentration as low as $10 \mathrm{pM}$, indicating the high sensitivity of our device. The control experiment using TAAR5 which does not respond to $\mathrm{CV}$ was also performed. We assembled the TAAR5-embedded nanodiscs (T5NDs), immobilized the T5NDs on floating electrode of CNT-FET and tested it with increasing concentration of CV (Figure S6). The CV was added up to $100 \mu \mathrm{M}$; however there is no meaningful response. Thus we can conclude the ONBN selectively binding to $\mathrm{CV}$ was effectively constructed. Moreover, for $\mathrm{CV}$, in sauerkraut, fish, cheese, fermented sausages and seasonings, maximum tolerable levels are about 4.2, 5.0, 5.3, 10.6 and $15.07 \mu \mathrm{M}$, respectively. ${ }^{31}$ The limit of detection of ONBN is about $10 \mathrm{pM}$ which is much lower than that of tolerance of $\mathrm{CV}$ in various foods. Therefore, our platform could be practically used to assessment of food quality. The plausible mechanism for this response is 
that the binding of protonated positively-charged $\mathrm{CV}$ molecules to TAAR13c increased the work function of the floating electrode. ${ }^{5}$ Thus, the binding of CV decreased the Schottky barrier for hole carriers in the device and increased the conductance of the ONBN device. ${ }^{32}$

Figure $4 \mathrm{~b}$ shows the graph for the normalized sensitivity $N$ of ONBNs at different concentrations of $\mathrm{CV}$. The normalized sensitivity was estimated by normalizing the sensor sensitivity $\left(\left|\Delta G / G_{0}\right|\right)$ with respect to its maximum sensitivity value. ${ }^{33-36}$ The measured response curves can be analyzed using the model based on the Langmuir isotherm theory as previous studies. ${ }^{37}$ In this model, the density $C_{s}$ of CV molecules bound to the T13NDs on an ONBN can be written as

$$
C_{S}=\frac{C_{s \max } C}{1 / K+C}
$$

where $C$ and $K$ represent the concentration of odorant in a solution and an equilibrium constant between odorant and T13NDs, respectively. $C_{s_{\text {max }}}$ is the density of T13NDs on the electrodes of an ONBN. The binding events between CV and T13NDs induced increased conductance. If we assume that a conductance change $\Delta G$ is linearly proportional to the number of bound $\mathrm{CV}$ molecules on T13NDs, a sensor sensitivity can be approximated as $\left|\Delta G / G_{0}\right| \sim k C_{s}$, where $k$ is a constant representing the response characteristics of an ONBN. Therefore, the sensor sensitivity could be written as

$$
\left|\Delta G / G_{0}\right|=k \frac{C_{s \max } C}{1 / K+C}
$$

As $C$ becomes very large, the sensor sensitivity $\left|\Delta G / G_{0}\right|$ could converge to $k \cdot C_{s} \max$. Thus the normalized sensitivity $N$ can be written as

$$
N=\frac{C}{1 / K+C}
$$


By fitting the measured data with this model, we could estimate the equilibrium constant $K$ between T13NDs and $\mathrm{CV}$ as $3.63 \times 10^{11} \mathrm{M}^{-1}$, which is a rather large compared with previously-reported values measured using cells or biological systems. This result implies that our ONBN device can detect target CV molecules with a much higher sensitivity than cells or biological systems. Moreover, these results demonstrate that our ONBN show the reproducible signals in repeated experiments.

Figure $4 \mathrm{c}$ shows the real-time response of an ONBN to various molecules containing amine functional groups. Here, we injected diaminodecane (DD), trimethylamine (TMA), ethanolamine (EA), glutamine (Glu) and CV molecules to the ONBN in series while measuring the sensor responses. DD and EA have the similar structures with CV, TMA has a fishy odor, and Glu is an amino acid. The structures of these molecules are presented in Supporting Information (Figure S2). Note that the ONBN device exhibited negligible sensor responses to non-target molecules even at a relatively high concentration of $1 \mu \mathrm{M}$. However, the conductance of the ONBN increased sharply by adding CV at a concentration of $1 \mathrm{nM}$, which is 1000 times lower concentration than that of non-target molecular species. This result clearly shows that our ONBN can selectively detect $\mathrm{CV}$ at a very low concentration even in the presence of high concentrations of similar molecular species. The amplitude of response was compatible with that in figure 4a. However, the noise level of this experiment was higher than previous one in figure $4 \mathrm{a}$. They are the same response in figure $4 \mathrm{a}$ and $4 \mathrm{c}$, but the shape of response could seem different because of the difference between their noise levels (signalto-noise ratio in figure $4 \mathrm{c}: \sim 4.3$ ).

Figure $4 \mathrm{~d}$ shows the responses of ONBNs to various real-sample solutions obtained from spoiled foods. Each tested food sample was prepared in HEPES buffer solution and filtered by ultrafiltration membranes (see METHODS section). The ONBN exhibited a higher 
response to samples from salmon and beef with a longer spoilage period. On the other hand, the samples from salmon with sorbic acid, one of the preservatives, exhibited decreased sensor responses, presumably due to the reduced spoilage of the salmon by sorbic acid. It indicates that our ONBN devices can be used to evaluate the quality of real-food samples quantitatively. Interestingly, the pork fat sample showed negligible sensor responses even after a long spoilage period. This is presumably because $\mathrm{CV}$, the target of our ONBN device, is the product of a decarboxylation of lysine which existed in salmon and beef at a large quantity, but not in pork fat. ${ }^{38}$ These results indicate our ONBN devices can be utilized to evaluate the quality of various real-food samples quantitatively under complex environmental conditions.

\section{CONCLUSIONS}

In conclusion, we developed a highly-stable ND-based bioelectronic nose for the detection of $\mathrm{CV}$. The NDs were successfully constructed with TAAR13c produced in E. coli. TAAR13c receptors in ND keep the native receptors properties and permit highly sensitive and selective detections of CV. Especially, the immobilization of T13NDs in a desired orientation on floating electrodes via linker molecules enabled a high density of recognition elements and active binding pockets in our ONBN devices, which results in its high sensitivity and selectivity. Furthermore, an ONBN measured CV quantitatively in real-food samples with different spoilage periods. These results show that our ONBN device based on high-quality GPCR-conjugated FETs is attractive technique for the detection of food spoilage and has a great potential for other practical applications such as detection of corpses. 


\section{METHODS}

Materials. Zebrafish (Danio rerio) cDNA was donated by Prof. Hyunsook Lee (Seoul National University). Palmitoyloleoylphosphatidylcholine (POPC) and palmitoyloleoylphosphatidylglycerol (POPG) were purchased from Avanti Polar Lipids (USA). Sodium cholate and HEPES were purchased from Sigma-Aldrich (USA).

Gene cloning of ApoA-I and TAAR13c. The ApoA-I gene was designed so that it contained 6xHis and stop codon gene, and it was amplified by PCR with primers (5' CAC CAG GAG ATA TAC ATA TGA AAG CTG CGG TGC TGA CC 3', 5' CTA GTG GTG GTG GTG GTG GTG CTG GGT GTT GAG CTT CTT AGT GTA 3') using human genomic DNA. The TAAR13c gene was amplified by PCR with primers (5' CAC CAG GAG ATA TAC ATA TGA TGC CCT TTT GCC ACA AT 3', 5' TGA ACT CAA TTC CAA AAA TAA TTT ACA C 3') using the zebrafish cDNA. Amplified PCR products were inserted into the pET-DEST42 bacterial expression vector (Invitrogen, USA) using a gateway cloning system (Invitrogen, USA). The TAAR13c gene was also cloned into pcDNA3 mammalian expression vector using an amplified PCR product (primer; 5' ATG AAT TCA TGG ATT TAT CAT CAC AAG AAT 3', 5' ATC TCG AGT CAA ACC GTA AAT AAA TTG ATA 3').

Expression of TAAR13c in HEK-293 cell. Human embryonic kidney (HEK)-293 cells were cultured in Dulbecco's Modified Eagles Medium (DMEM) (HyClone, USA) supplemented with $1 \%$ penicillin, $1 \%$ streptomycin (Gibco, USA) and 10\% Fetal Bovine Serum (FBS) (Gibco, USA) at $37{ }^{\circ} \mathrm{C}$ under $5 \% \mathrm{CO}_{2}$. The transfection was performed with Lipofectamine3000 (Invitrogen, USA) following the manufacturer's protocol. The cells were transfected with Liopofectamine3000 DNA mixture containing TAAR13c, pCRE-Luc, pSV40-RL, $\mathrm{G}_{\text {oolf }}$ and RTP1S. The cells were harvested with phosphate-buffed saline, (PBS, $\mathrm{pH} 7.4$ ) and then disrupted by sonication (2 s on/off, $2 \mathrm{~min}$ ) (Sonics Vibracell, USA). 
Dual luciferase reporter assay. The Dual-Glo Luciferase Assay System (Promega, USA) was introduced to analyze biological activity of TAAR13c. The transfected cells were stimulated with $50 \mu \mathrm{L}$ of serum-free DMEM for $30 \mathrm{~min}$ and then incubated with various amine species for $4 \mathrm{~h}$. The luminescence activity was measured with a Spark 10M multimode microplate reader (TECAN, USA). The normalized luciferase activity was calculated with the formula [CRE/Renilla(N) - CRE/Renilla(0)]/[CRE/Renilla(FSK) - CRE/Renilla(0)]. A no amine solution was used as a negative control, and the $10 \mu \mathrm{M}$ forskolin (FSK) was used as a positive control.

Purification of ApoA-I. BL21(DE3) E. coli cells bearing the pET-DEST42/ApoA-I construct were cultured in $1 \mathrm{~L}$ Luria-Bertani (LB) medium $\left(+50 \mu \mathrm{g} / \mathrm{mL}\right.$ ampicillin) at $37^{\circ} \mathrm{C}$. When the cells were grown until the $\mathrm{OD}_{600}$ value reached 0.5, the overexpression of ApoA-I was induced by the addition of isopropyl thiogalactoside (IPTG) to a final concentration of 1 $\mathrm{mM}$. After $3 \mathrm{~h}$, the cells were centrifuged $\left(7000 \mathrm{~g}, 20 \mathrm{~min}, 4{ }^{\circ} \mathrm{C}\right)$, resuspended in a lysis buffer (20 mM Tris-HCl, $0.5 \mathrm{M} \mathrm{NaCl}, 20 \mathrm{mM}$ imidazole, $\mathrm{pH} 8.0$ ) and disrupted by sonication (5 s on/off, $5 \mathrm{~min}$ ). The cell lysate was centrifuged at $12,000 \mathrm{~g}$ for $30 \mathrm{~min}$ at $4{ }^{\circ} \mathrm{C}$. The ApoAI in the supernatant was collected and loaded to HisTrap HP column (GE Healthcare, Sweden) through FPLC (GE Healthcare). The column was washed with washing buffer (20 mM Tris$\mathrm{HCl}, 50 \mathrm{mM}$ imidazole, $0.5 \mathrm{M} \mathrm{NaCl}, \mathrm{pH} 8.0$ ), and the ApoA-I was eluted with the elution buffer (20 mM Tris-HCl, $400 \mathrm{mM}$ imidazole, $0.5 \mathrm{M} \mathrm{NaCl}, \mathrm{pH} 8.0$ ) and dialyzed against HEPES buffer I (20 mM HEPES-NaOH, $100 \mathrm{mM} \mathrm{NaCl,} 20 \mathrm{mM}$ cholate, 1mM EDTA, pH 8.0) using HiTrap HP desalting column (GE Healthcare, Sweden). The proteins were stored at $4{ }^{\circ} \mathrm{C}$ until used.

Purification of TAAR13c. The BL21 (DE3) cells were transformed with the pETDEST42/TAAR13c vector and cultured in LB medium $\left(+50 \mu \mathrm{g} / \mathrm{mL}\right.$ ampicillin) at $37^{\circ} \mathrm{C}$ until 
the $\mathrm{OD}_{600}$ value reached 0.5 . The expression of TAAR13c was induced by the addition of 1 mM IPTG, and the cells were incubated for $4 \mathrm{~h}$. The cells were centrifuged (7000 g, $20 \mathrm{~min}$, $4{ }^{\circ} \mathrm{C}$ ), and pellets were resuspended in PBS containing $2 \mathrm{mM}$ EDTA. The cells were disrupted by sonication ( $5 \mathrm{~s} \mathrm{on} / \mathrm{off}, 5 \mathrm{~min}$ ) and centrifuged $\left(12000 \mathrm{~g}, 4{ }^{\circ} \mathrm{C}, 20 \mathrm{~min}\right)$. The sonication and centrifugation were repeated, and the pellet of the sample was solubilized in solubilization buffer $(0.1 \mathrm{M}$ Tris- $\mathrm{HCl}, 20 \mathrm{mM}$ sodium dodecyl sulfate (SDS), $100 \mathrm{mM}$ dithiothreitol (DTT), $1 \mathrm{mM}$ EDTA, $\mathrm{pH} 8.0$ ) at $25^{\circ} \mathrm{C}$. The solubilized proteins were dialyzed against $0.1 \mathrm{M}$ sodium phosphate ( $\mathrm{pH} 8.0$ ) containing $10 \mathrm{mM}$ SDS using a 10K MWCO dialysis cassette (Thermo Scientific, USA), filtered with a $0.2 \mu \mathrm{m}$ bottle top filter (Thermo Scientific, USA), and then applied to HisTrap HP column equilibrated in $0.1 \mathrm{M}$ sodium phosphate $(\mathrm{pH}$ 8.0) containing $10 \mathrm{mM}$ SDS. The column was successively washed with gradient ( $\mathrm{pH} 8.0$ to 7.0 ) using washing buffer (0.1 M sodium phosphate, $10 \mathrm{mM}$ SDS), and the TAAR13c was eluted with the same buffer at $\mathrm{pH}$ 6.0. The eluted protein was dialyzed against the HEPES buffer II (20 mM HEPES-NaOH, $100 \mathrm{mM} \mathrm{NaCl}, 25 \mathrm{mM}$ cholate, $1 \mathrm{mM}$ EDTA, $\mathrm{pH}$ 8.0). The purified TAAR13c was analyzed by SDS-PAGE and western blot analysis.

Western blot analysis and total protein assays. All protein samples $(20 \mu \mathrm{L})$ were analyzed by SDS-PAGE and western blot analysis. Western blot analysis was performed using anti-FLAG rabbit Ab (Cell Signaling Technology, USA), anti-His-probe mouse Ab (Santa Cruz Biotechnology, USA) and anti-V5 epitope mouse Ab (Santa Cruz Biotechnology, USA) as primary Ab. HRP-conjugated anti-rabbit Ab (Millipore, USA) and HRP-conjugated anti-mouse $\mathrm{Ab}$ (Milipore, USA) were used as a secondary Ab. Luminata Forte western HRP substrate (Millipore, USA) was also used. The protein concentration was measured using BCA assay kit (Pierce, IL, USA). 
Assembly of T13NDs. POPC and POPG were used in combination at a 1:1 molar ratio to mimic the native membrane-like environment. Lipids were dried using nitrogen gas from a chloroform solution and vacuumed for $1 \mathrm{~h}$ to remove residual chloroform. The lipids were solubilized in HEPES buffer II, added to purified TAAR13c protein, and then incubated for $10 \mathrm{~min}$ in ice. After that the ApoA-I was added, and the mixtures were incubated for $2 \mathrm{~h}$ at 4 ${ }^{\circ} \mathrm{C}$ with stirring. The final concentrations of the mixture were $1 \mu \mathrm{M}$ TAAR13c, $100 \mu \mathrm{M}$ ApoA-I, $8 \mathrm{mM}$ lipids and 25mM detergent. Next, Bio-Beads (Bio-Rad, USA) were added to this mixture to remove the detergents with overnight agitation. Finally, the mixture was applied to a size exclusion chromatography (SEC) (Superdex 200 Increase 10/300 GL, GE Healthcare, USA) to remove unbound proteins. The column was equilibrated with HEPES buffer III (20 mM HEPES-NaOH, $100 \mathrm{mM} \mathrm{NaCl}, 1 \mathrm{mM}$ EDTA, $\mathrm{pH} 8.0)$ and the $500 \mu \mathrm{L}$ of sample was loaded in injecting loops at a flow rate of $0.5 \mathrm{~mL} / \mathrm{min}$ using FPLC. The peak fractions were collected and the purified T13NDs was stored at $4{ }^{\circ} \mathrm{C}$ before characterization steps.

Analyses of T13NDs. The sizes of NDs were analyzed using a dynamic light scattering spectrophotometer (DLS) (DLS-7000, Japan). The T13NDs was also confirmed by using a SUPRA 55VP field-emission scanning electron microscope (FE-SEM) (Carl Zeiss, Germany). The intrinsic fluorescence of T13NDs was measured in real-time using a LS 55 luminescence spectrometer (Perkin Elmer, USA) (excitation $290 \mathrm{~nm}$; emission $340 \mathrm{~nm}$ ). The real-time intrinsic fluorescence of TAAR13c was measured using 1-10 mM of various amines.

Immobilization of T13NDs on floating electrode of CNT-FET using V5 Ab. Halffragment $\mathrm{Ab}$ was generated by 2-MEA reagent. Channels were incubated in $0.5 \mathrm{M}$ N-acetyl cysteine for $10 \mathrm{~min}$. Subsequently, channels were gently rinsed in deionized (DI) water. The thiol-functionalized channels were dipped in half-fragment V5 Ab solution at $37{ }^{\circ} \mathrm{C}$ for $1 \mathrm{~h}$. 
Finally, the V5-functionalized floating electrodes were incubated in T13ND solution for 30 min at room temperature. For fluorescence microscopy, V5 Ab with Dylight 488 solution (Thermo Scientific, USA) was diluted with PBS (0.02 mg Ab/ml) and applied to the channel for $1 \mathrm{~h}$. The droplet was then washed with PBS solution, instead of the final T13NDs incubation step. In the case of AFM measurement, we exchanged the buffer solution for DI water.

Preparation of tested samples. All the tested amines were prepared at $1 \mathrm{mM}$ in $100 \%$ DMSO, and serially diluted 1:10 with HEPES buffer. Fresh foods (salmon (Norway), beef (Korea), pork fat (Korea)) were purchased from a local grocery store. Each food sample (100 mg) was soaked in HEPES buffer solution (1mL) and incubated at $37{ }^{\circ} \mathrm{C}$ for 4 weeks to prepare the real samples. The spoiled foods samples were collected weekly ( 0 - 4 weeks $)$ and kept in microtubes at $-80{ }^{\circ} \mathrm{C}$. The frozen samples were thawed at once just before the electrical measurements. The sample solutions were diluted to $1 / 1000$ and filtered by 100,000 nominal molecular weight cut-off (NMWCO) ultrafiltration membranes (Ultracel YM-100, Millipore, USA) with centrifugation at $1000 \mathrm{~g}$ for $10 \mathrm{~min}$ to remove floating matter in the sample solutions.

Measurement of gate profiles and noise characteristics. The gate profiles were measured by a semiconductor analyzer (4200-SCS, Keithley, USA). A drain-source bias voltage of 0.1 $\mathrm{V}$ was maintained during measuring the characteristics. When a DI water droplet of $10 \mu \mathrm{L}$ was placed on the channel of an ONBN, the gate bias voltage was applied through the DI water droplet and swept from $-0.5 \mathrm{~V}$ to $0.5 \mathrm{~V}$. We utilized a fast Fourier-transform network analyzer (SR 770) for the measurement of the noise characteristics. The bias voltage of $0.1 \mathrm{~V}$ was applied to the ONBN sensor. 


\section{ASSOCIATED CONTENT}

Supporting Information: Additional information about experimental details, NDs purification, MSP preparation, CNT fabrication and real sample data. The Supporting Information is available free of charge via the Internet at http://pubs.acs.org.

\section{AUTHOR INFORMATION}

\section{Corresponding Authors}

Prof. T. H. Park: Tel: +82-2-880-1874; Fax: +82-2-857-9348; E-mail: thpark@ snu.ac.kr

Prof. S. Hong: Tel: +82-2-880-1343; Fax: +82-2-884-5572; E-mail: seunghun@snu.ac.kr

\section{Author Contributions}

These authors contributed equally to this work.

\section{ACKNOWLEDGMENTS}

We thank Prof. Hyunsook Lee (Seoul National University) for donating the zebrafish (Danio rerio) cDNA. This research was supported by the National Research Foundation funded by the Korean government (MSIP) (2016945295). This project has received funding from the European Research Council (ERC) under the European Union's Horizon 2020 research and innovation programme (grant agreement No 682286). S.H. acknowledges the support the BioNano Health-Guard Research Center and NRF grant funded by MSIP (HGUARD 2013M3A6B2078961, 2014M3A7B4051591). This work was also supported by the Korea Basic Science Institute (D37410). 


\section{Figure Captions}

Figure 1. Schematic diagram of an ONBN. This figure illustrates the ONBN and possible sensor response curves of an ONBN. T13NDs were successfully immobilized on floating electrodes of a CNT-FET, which leads to a high-performance ONBN. The ONBN could selectively discriminate spoiled foods from fresh samples.

Figure 2. Characterization of TAAR13c expressed in HEK-293 cells and high-quality TAAR13c-embedded nanodiscs (T13NDs). The purified TAAR13c in NDs was produced in E. coli. (a) Dose-dependent response of TAAR13c to the CV $\left({ }^{*} \mathrm{p}<0.05,{ }^{* *} \mathrm{p}<0.01,{ }^{* * *} \mathrm{p}<0.001\right)$ $(n=3)$. The error bars represent the standard error of the mean. (b) Selectivity of TAAR13c with various amines (HA, hydroxylamine; EA, ethanolamine; PT, putrescine; $\mathrm{CV}$, cadaverine; DD, diaminodecane; TMA, trimethylamine; TEA, trimethylamine; ThiA, thiamine; TryA, tryptamine; Glu, glutamine) $(\mathrm{n}=3)$. The error bars represent the standard error of the mean. (c) SDS-PAGE analysis of purified TAAR13c produced in E. coli. The western blot analysis was performed with V5 Ab. (d) DLS size distribution of optimized T13NDs. (e) FE-SEM image of T13NDs. (f) Real-time tryptophan fluorescence of T13NDs with increasing concentration of the CV. (g) Selective response of T13NDs to CV measured by real-time tryptophan fluorescence.

Figure 3. Characteristics of an oriented NDs-functionalized CNT-FET with floating electrodes. (a) Fluorescence image and the intensity profile of V5 Ab-functionalized floating electrodes of a CNT-FET. The images represent the functionalized V5 Ab on floating electrodes of the ONBN. (b) Liquid AFM images and the sectional height profile of an ONBN. The AFM images show the immobilized-ND structures on the floating electrodes of ONBN. The height graph indicates that the immobilized-NDs had sizes similar to those 
reported previously. (c) Gate profiles of ONBNs with or without NDs. These are typical ptype semiconductor characteristics. (d) Electrical noise characteristics of an ONBN at a frequency domain exhibiting typical 1/f characteristics.

Figure 4. Responses of ONBN devices to various samples. (a) Real-time response of an ONBN to the various concentrations of $\mathrm{CV}$. The additions of $\mathrm{CV}$ solution induced the increase of conductance of the ONBN in dose-dependent manner. (b) Dose-dependent response curve of the ONBN to $\mathrm{CV}(\mathrm{n}=3)$. The error bars represent the standard error of the mean. By fitting the data using Eq. (3), the equilibrium constant $K$ was estimated as $3.63 \times 10^{11} \mathrm{M}^{-1}$. (c) Real-time response of an ONBN to various molecular species with amine functional groups. Non-target molecules caused negligible conductance changes of the ONBN. (d) Comparison of the sensor responses of ONBN devices to different spoiled food samples $(n=3)$. The error bars represent the standard error of the mean. As the spoilage period for salmon and beef increased, the ONBN devices exhibited higher sensor responses. 


\section{REFERENCES}

1. Siehler, S. Cell-Based Assays in GPCR Drug Discovery. Biotechnol. J. 2008, 3, 471483.

2. Drews, J. Drug Discovery: A Historical Perspective. Science 2000, 287, 1960-1964.

3. Hussain, A.; Saraiva, L. R.; Ferrero, D. M.; Ahuja, G.; Krishna, V. S.; Liberles, S. D.; Korsching, S. I. High-Affinity Olfactory Receptor for the Death-Associated Odor Cadaverine. Proc. Natl. Acad. Sci. U. S. A. 2013, 110, 19579-19584.

4. Liberles, S. D. Trace Amine-Associated Receptors Are Olfactory Receptors in Vertebrates. Ann. N. Y. Acad. Sci. 2009, 1170, 168-172.

5. Sharma, K.; Ahuja, G.; Hussain, A.; Balfanz, S.; Baumann, A.; Korsching, S. I. Elimination of a Ligand Gating Site Generates a Supersensitive Olfactory Receptor. Sci. Rep. 2016, 6, 28359.

6. Liberles, S. D.; Buck, L. B. A Second Class of Chemosensory Receptors in the Olfactory Epithelium. Nature 2006, 442, 645-650.

7. Liberles, S. D. Trace Amine-Associated Receptors: Ligands, Neural Circuits, and Behaviors. Curr. Opin. Neurobiol. 2015, 34, 1-7.

8. Grisshammer, R.; Tate, C. G. Overexpression of Integral Membrane Proteins for Structural Studies. Q. Rev. Biophys. 1995, 28, 315-422.

9. Gafvelin, G.; von Heijne, G. Topological "Frustration" in Multispanning E. Coli Inner Membrane Proteins. Cell 1994, 77, 401-412. 
10. Yang, H.; Song, H.; Ahn, S.; Park, T. H. Purification and Functional Reconstitution of Human Olfactory Receptor Expressed in Escherichia Coli. Biotechnol. Bioproc. E. 2015, $20,423-430$.

11. Denisov, I. G.; Sligar, S. G. Nanodiscs for Structural and Functional Studies of Membrane Proteins. Nat. Struct. Mol. Biol. 2016, 23, 481-486.

12. Serebryany, E.; Zhu, G. A.; Yan, E. C. Artificial Membrane-Like Environments for in Vitro Studies of Purified G-Protein Coupled Receptors. Biochim. Biophys. Acta 2012, $1818,225-233$.

13. Goldsmith, B. R.; Mitala, J. J.; Josue, J.; Castro, A.; Lerner, M. B.; Bayburt, T. H.; Khamis, S. M.; Jones, R. A.; Brand, J. G.; Sligar, S. G.; Luetje, C. W.; Gelperin, A.; Rhodes, P. A.; Discher, B. M.; Johnson, A. T. Biomimetic Chemical Sensors Using Nanoelectronic Readout of Olfactory Receptor Proteins. ACS Nano 2011, 5, 5408-5416.

14. Glück, J. M.; Koenig, B. W.; Willbold, D. Nanodiscs Allow the Use of Integral Membrane Proteins as Analytes in Surface Plasmon Resonance Studies. Anal. Biochem. 2011, 408, 46-52.

15. Lee, J.; Lee, H.; Kim, T.; Jin, H. J.; Shin, J.; Shin, Y.; Park, S.; Khang, Y.; Hong, S. Floating Electrode Transistor Based on Purified Semiconducting Carbon Nanotubes for High Source-Drain Voltage Operation. Nanotechnology 2012, 23, 085204.

16. Lee, M.; Jung, J. W.; Kim, D.; Ahn, Y. J.; Hong, S.; Kwon, H. W. Discrimination of Umami Tastants Using Floating Electrode-Based Bioelectronic Tongue Mimicking Insect Taste Systems. ACS Nano 2015, 9, 11728-11736. 
17. Karyakin, A. A.; Presnova, G. V.; Rubtsova, M. Y.; Egorov, A. M. Oriented Immobilization of Antibodies onto the Gold Surfaces Via Their Native Thiol Groups. Anal. Chem. 2000, 72, 3805-3811.

18. Oh, J.; Yoo, G.; Chang, Y. W.; Kim, H. J.; Jose, J.; Kim, E.; Pyun, J. C.; Yoo, K. H. A Carbon Nanotube Metal Semiconductor Field Effect Transistor-Based Biosensor for Detection of Amyloid-Beta in Human Serum. Biosens. Bioelectron. 2013, 50, 345-350.

19. Lo, Y. S.; Nam, D. H.; So, H. M.; Chang, H.; Kim, J. J.; Kim, Y. H.; Lee, J. O. Oriented Immobilization of Antibody Fragments on Ni-Decorated Single-Walled Carbon Nanotube Devices. ACS Nano 2009, 3, 3649-3655.

20. Zhuang, H.; Matsunami, H. Evaluating Cell-Surface Expression and Measuring Activation of Mammalian Odorant Receptors in Heterologous Cells. Nat. Protoc. 2008, 3, 1402.

21. Song, H. S.; Lee, S. H.; Oh, E. H.; Park, T. H. Expression, Solubilization and Purification of a Human Olfactory Receptor from Escherichia Coli. Curr. Microbiol. 2009, 59, 309-314.

22. Imura, T.; Tsukui, Y.; Taira, T.; Aburai, K.; Sakai, K.; Sakai, H.; Abe, M.; Kitamoto, D. Surfactant-Like Properties of an Amphiphilic A-Helical Peptide Leading to Lipid Nanodisc Formation. Langmuir 2014, 30, 4752-4759.

23. Michalke, K.; Huyghe, C.; Lichiere, J.; Graviere, M. E.; Siponen, M.; Sciara, G.; Lepaul, I.; Wagner, R.; Magg, C.; Rudolph, R.; Cambillau, C.; Desmyter, A. Mammalian G Protein-Coupled Receptor Expression in Escherichia Coli: II. Refolding and Biophysical 
Characterization of Mouse Cannabinoid Receptor 1 and Human Parathyroid Hormone Receptor 1. Anal. Biochem. 2010, 401, 74-80.

24. Son, M.; Kim, D.; Ko, H. J.; Hong, S.; Park, T. H. A Portable and Multiplexed Bioelectronic Sensor Using Human Olfactory and Taste Receptors. Biosens. Bioelectron. 2017, 87, 901-907.

25. Whorton, M. R.; Jastrzebska, B.; Park, P. S.; Fotiadis, D.; Engel, A.; Palczewski, K.; Sunahara, R. K. Efficient Coupling of Transducin to Monomeric Rhodopsin in a Phospholipid Bilayer. J. Biol. Chem. 2008, 283, 4387-4394.

26. Allen, M. J.; Hud, N. V.; Balooch, M.; Tench, R. J.; Siekhaus, W. J.; Balhorn, R. Tip-Radius-Induced Artifacts in AFM Images of Protamine-Complexed DNA Fibers. Ultramicroscopy 1992, 42-44, 1095-1100.

27. Schwarz, U. D.; Haefke, H.; Reimann, P.; Guntherodt, H. J. Tip Artifacts in Scanning Force Microscopy. J. Microsc. (Oxford, U. K.) 1994, 173, 183-197.

28. Venkatakrishnan, A. J.; Deupi, X.; Lebon, G.; Tate, C. G.; Schertler, G. F.; Babu, M. M. Molecular Signatures of G-Protein-Coupled Receptors. Nature 2013, 494, 185-194.

29. Kim, J.; Hong, D.; Lee, H.; Shin, Y.; Park, S.; Khang, Y.; Lee, M.; Hong, S. Large Scale Assembly of Pristine Semiconducting Carbon Nanotube Network-Based Devices Exhibiting Intrinsic Characteristics. J. Phys. Chem. C 2013, 117, 19721-19728.

30. Soliveres, S.; Gyani, J.; Delseny, C.; Hoffmann, A.; Pascal, F. V F Noise and Percolation in Carbon Nanotube Random Networks. Appl. Phys. Lett. 2007, 90, 082107. 
31. Rauscher-Gabernig, E.; Gabernig, R.; Brueller, W.; Grossgut, R.; Bauer, F.; Paulsen, P. Dietary Exposure Assessment of Putrescine and Cadaverine and Derivation of Tolerable Levels in Selected Foods Consumed in Austria. Eur. Food Res. Technol. 2012, 235, 209-220.

32. Kim, B.; Lee, J.; Namgung, S.; Kim, J.; Park, J. Y.; Lee, M. S.; Hong, S. DNA Sensors Based on CNT-FET with Floating Electrodes. Sens. Actuators, B 2012, 169, 182187.

33. Kim, T. H.; Song, H. S.; Jin, H. J.; Lee, S. H.; Namgung, S.; Kim, U.-k.; Park, T. H.; Hong, S. "Bioelectronic Super-Taster" Device Based on Taste Receptor-Carbon Nanotube Hybrid Structures. Lab Chip 2011, 11, 2262-2267.

34. Takagi, S.; Toko, K.; Wada, K.; Ohki, T. Quantification of Suppression of Bitterness Using an Electronic Tongue. J. Pharm. Sci. 2001, 90, 2042-2048.

35. Jin, H. J.; Lee, S. H.; Kim, T. H.; Park, J.; Song, H. S.; Park, T. H.; Hong, S. Nanovesicle-Based Bioelectronic Nose Platform Mimicking Human Olfactory Signal Transduction. Biosens. Bioelectron. 2012, 35, 335-341.

36. Kim, T. H.; Lee, S. H.; Lee, J.; Song, H. S.; Oh, E. H.; Park, T. H.; Hong, S. Single Carbon Atomic Resolution Detection of Odorant Molecules Using a Human Olfactory Receptor-Based Bioelectronic Bose. Adv. Mater. 2009, 21, 91-94.

37. Patnaik, P.; Dean, J. A. Dean's Analytical Chemistry Handbook. McGraw-Hill: New York, 2004.

38. Pipek, P.; Rohlík, B.-A.; Potůček, T.; Šimoniová, A. The Composition of Pork Lard as a Raw Material in Meat Production. Maso International 2012, 2, 115-119. 


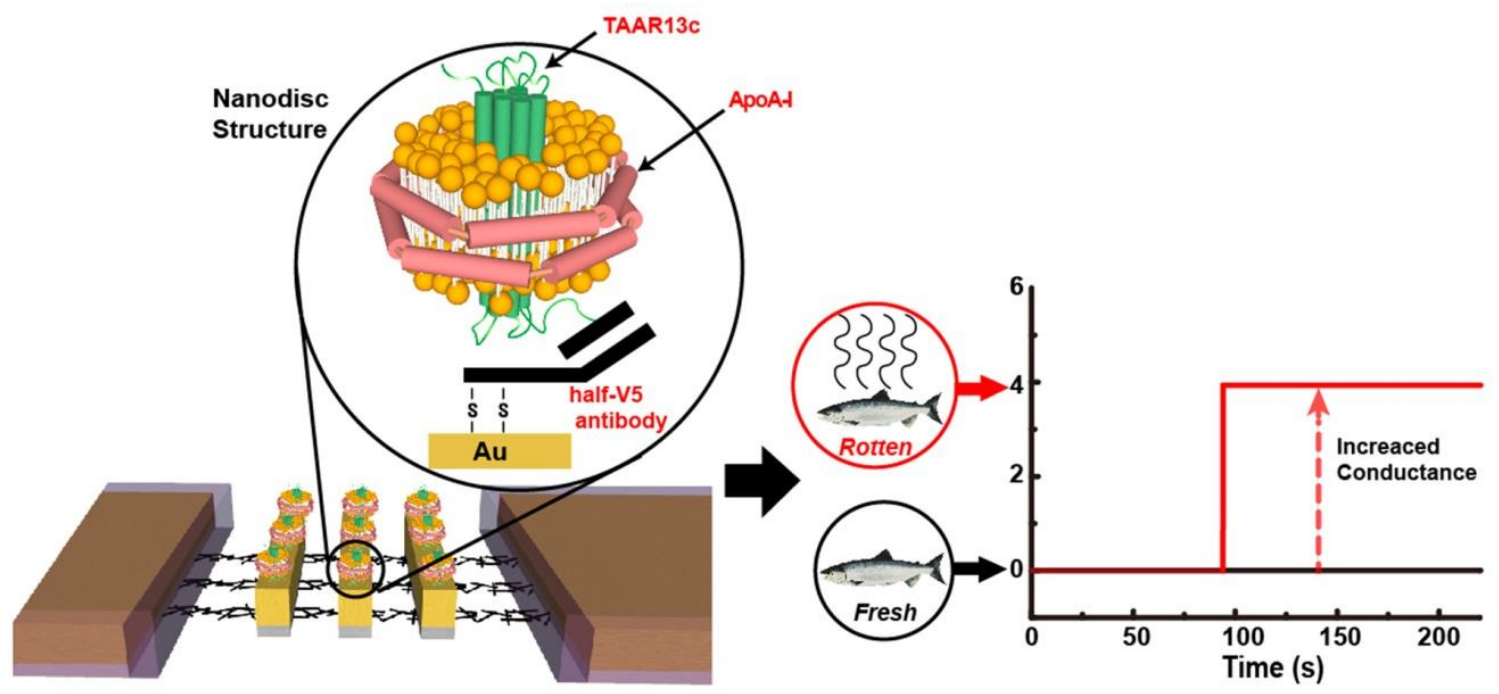

Figure 1 

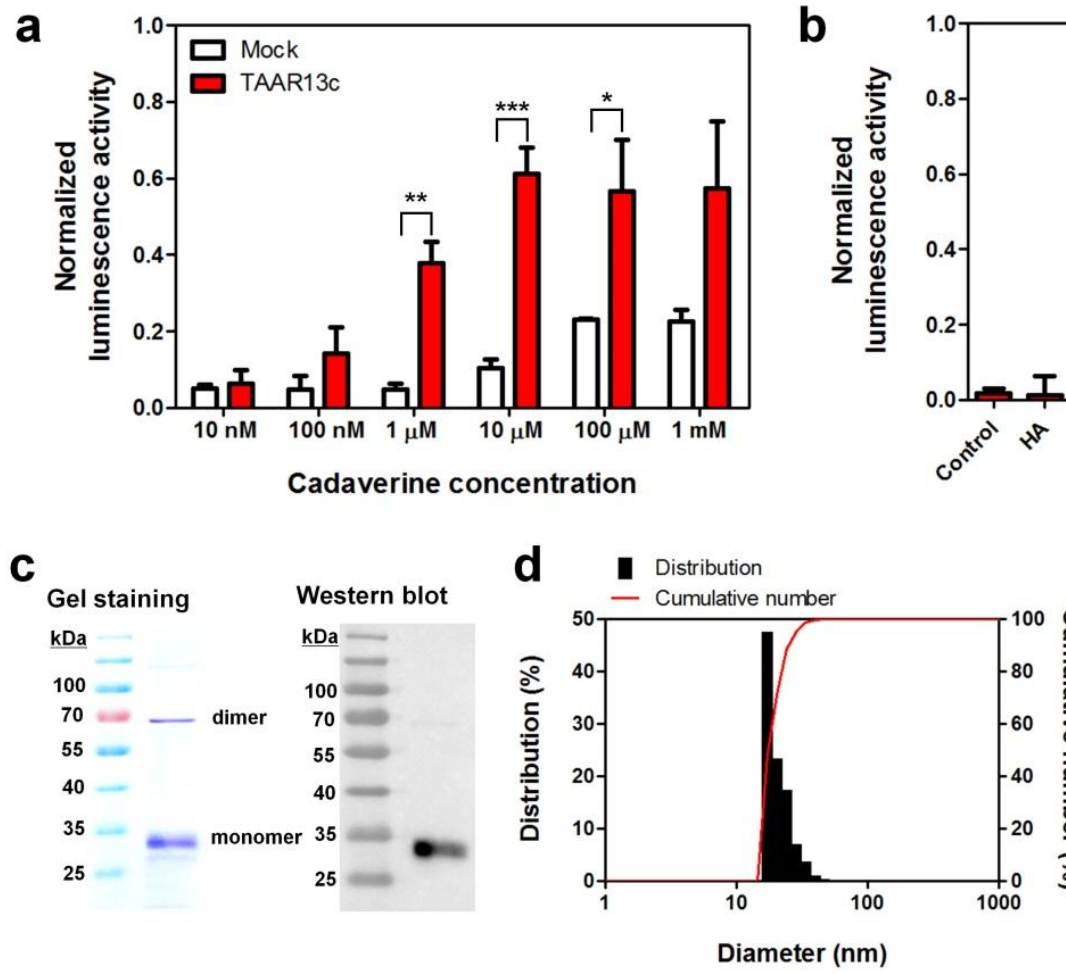

e
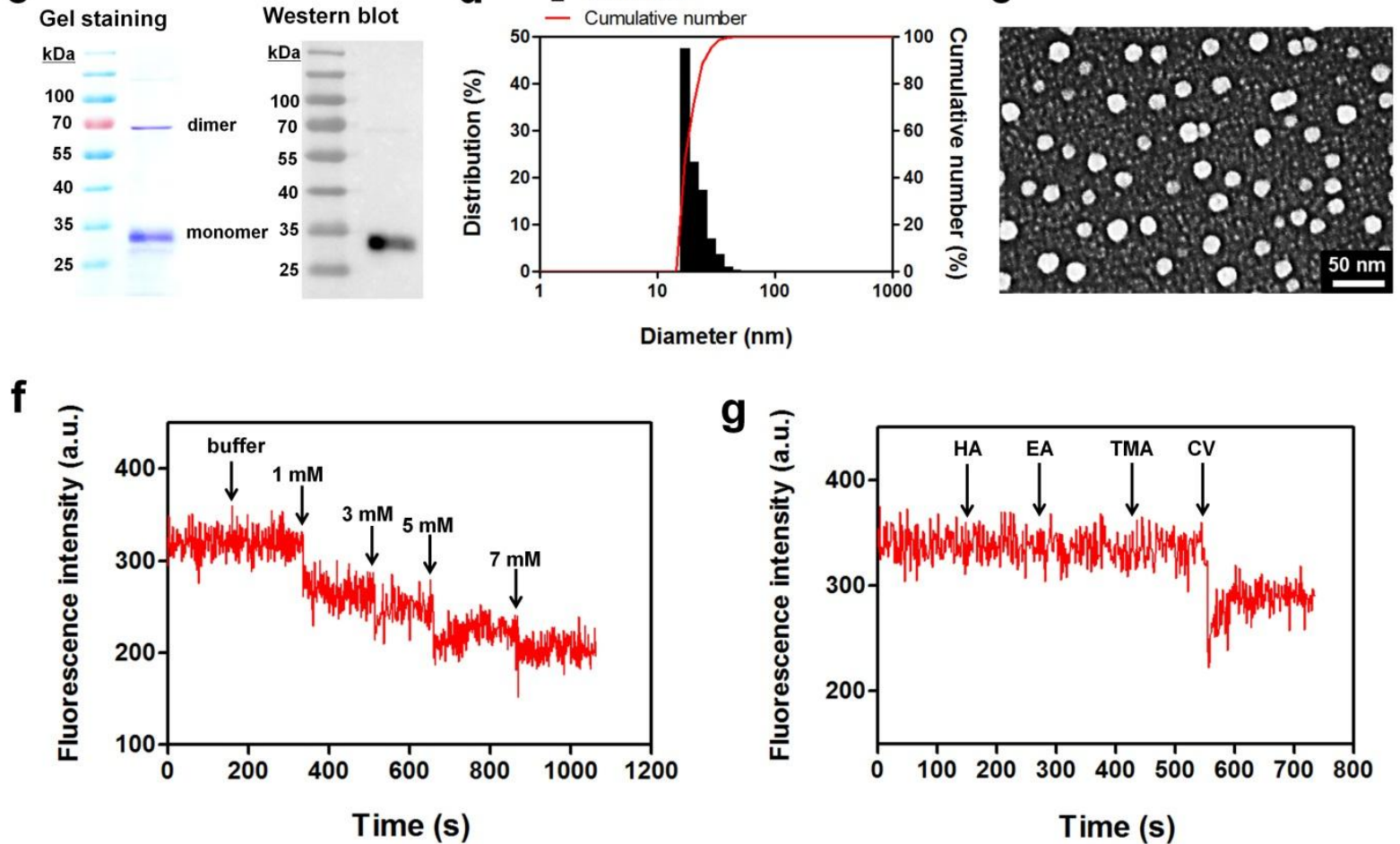

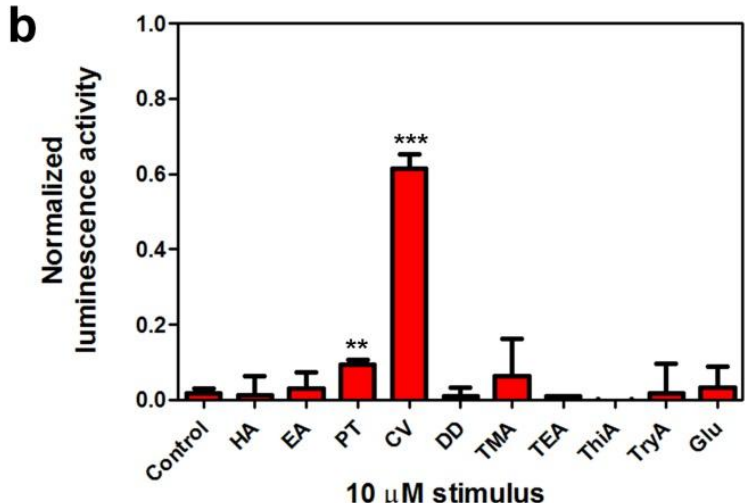
$10 \mu \mathrm{M}$ stimulus

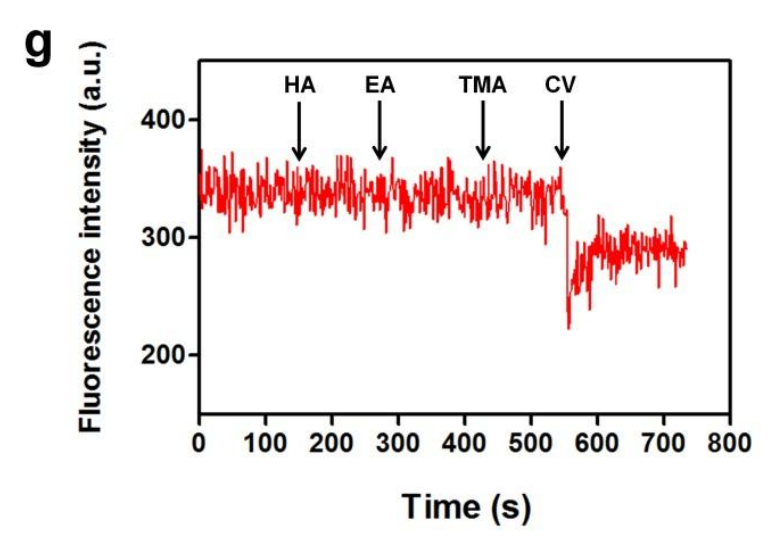

Figure 2 
a

C
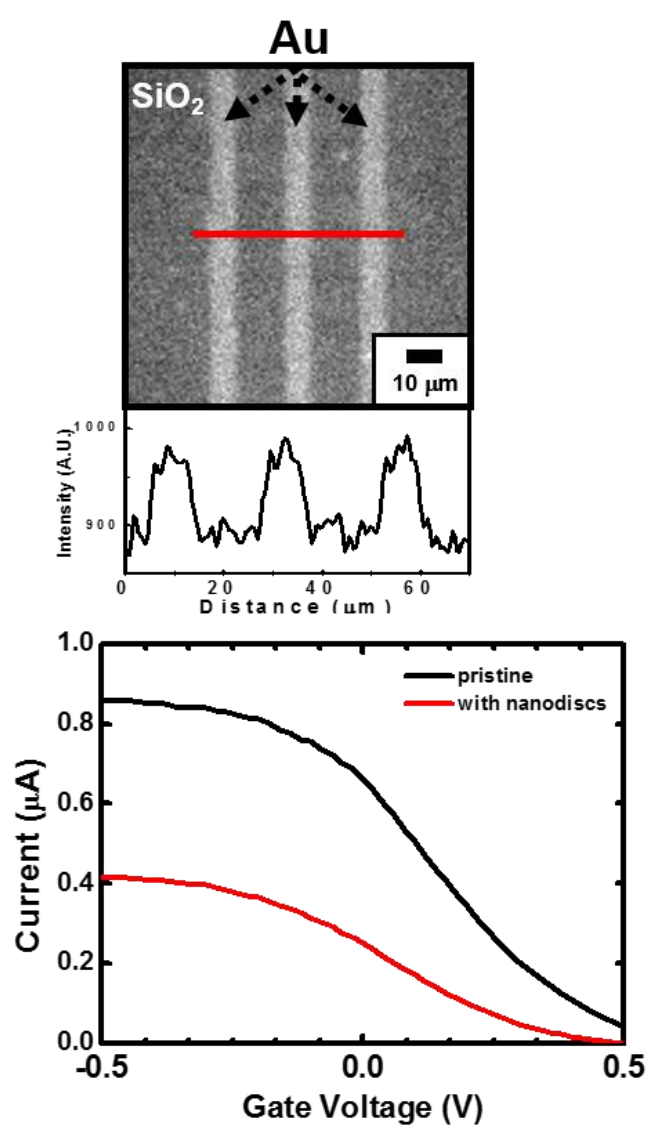

b

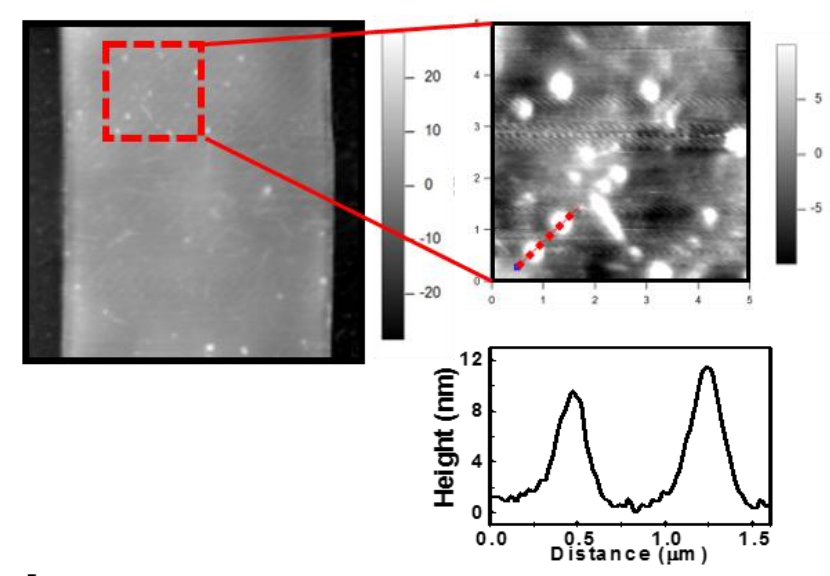

d

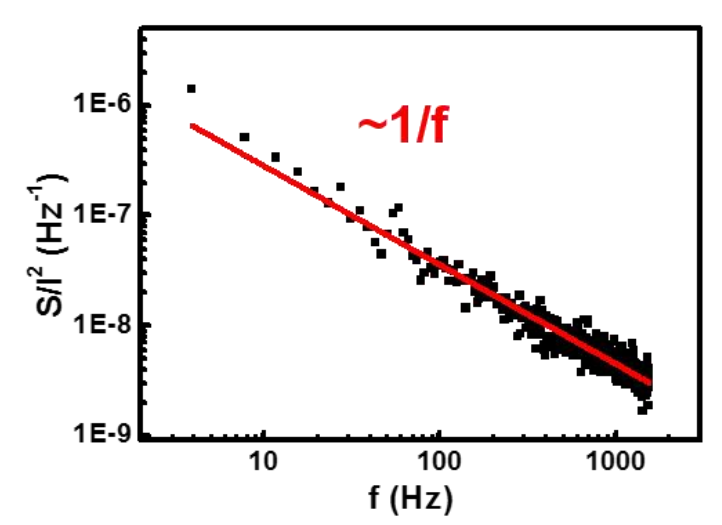

Figure 3 
a
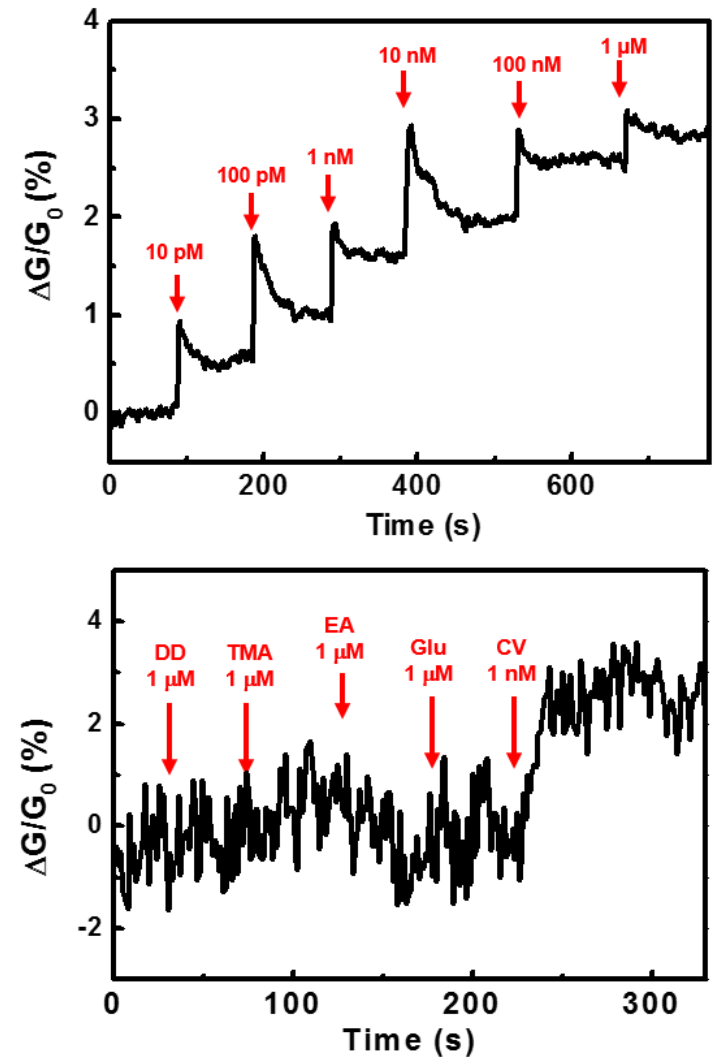

b

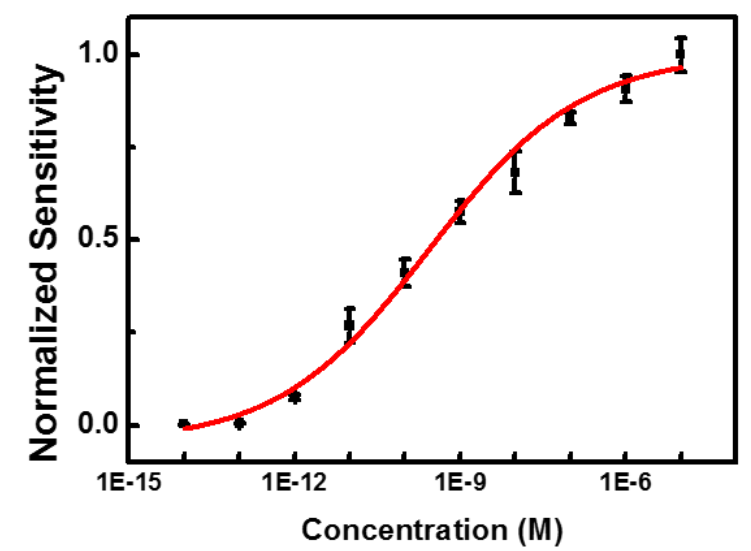

d

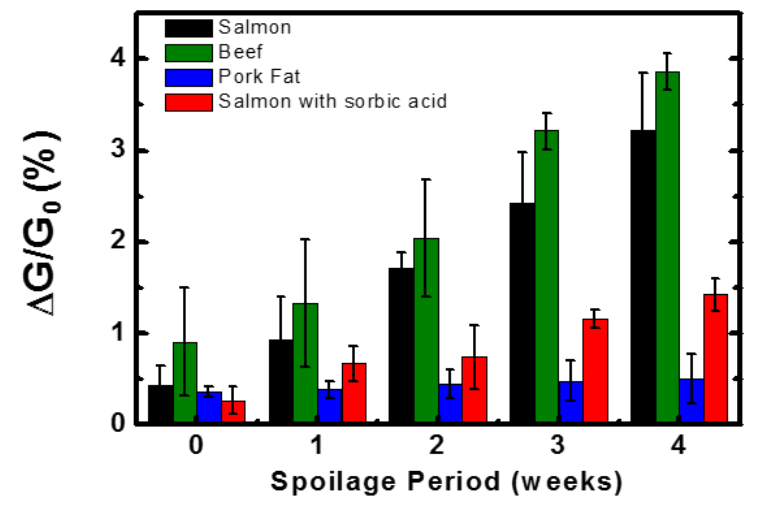

Figure 4 
Table of Contents (TOC)
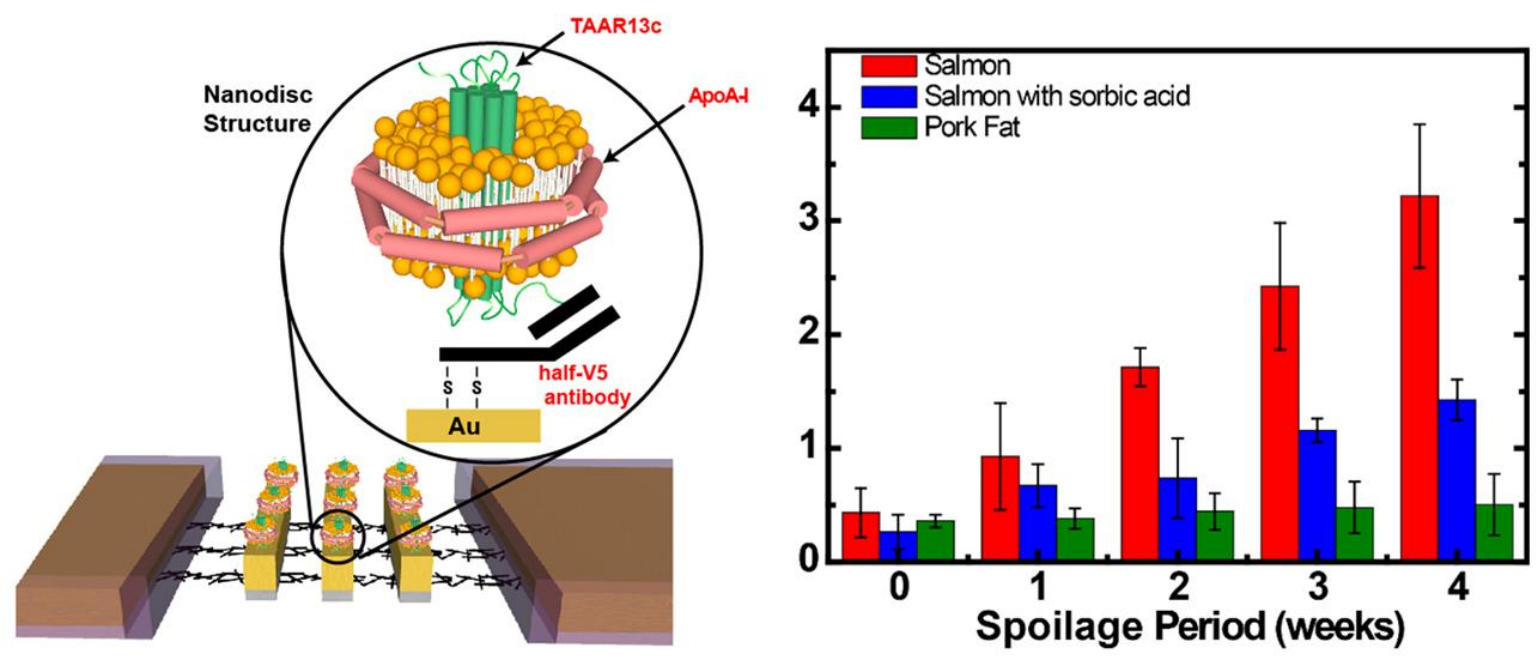